\title{
Role of Interfacial Tension for the Structure of PEP-PEO Polymeric Micelles. A Combined SANS and Pendant Drop Tensiometry Investigation
}

\author{
Reidar Lund, Lutz Willner,* Jörg Stellbrink, Aurel Radulescu, and Dieter Richter
}

Institut für Festkörperforschung, Forschungszentrum Jülich, 52425 Jülich, Germany

Received October 30, 2003; Revised Manuscript Received October 4, 2004

\begin{abstract}
We investigated the influence of interfacial tension, $\gamma$, on the micellization properties of a highly asymmetric poly(ethylene-co-propylene)-poly(ethylene oxide) (PEP-PEO) block copolymer in mixed solvents consisting of water and dimethylformamide (DMF). Both are good solvents for PEO and nonsolvents for PEP but exhibit a large difference in $\gamma$ with respect to the insoluble core block. Micellar characteristics were obtained by small-angle neutron scattering (SANS) and subsequent fitting of a coreshell form factor to the scattering patterns. The curves are perfectly described by a hyperbolic density profile for the shell, $n(r) \sim r^{-4 / 3}$, indicating a starlike structure of the micelles. The aggregation numbers of the micelles decrease with increasing $\mathrm{DMF}$-water ratio from $P=120$ in pure water to nonaggregated chains in pure DMF. Corresponding interfacial tensions were determined by pendant drop tensiometry using a PEP homopolymer of equal molar mass. A correlation of $P$ with $\gamma$ reveals a power law dependence, $P \sim \gamma^{6 / 5}$, in accordance with the scaling prediction of Halperin for starlike micelles. Additionally, it was found that the addition of DMF leads to a considerable decrease in the micelle radii, which cannot be explained by the decrease in $P$ alone. Measurements of the second virial coefficients, $A_{2}$, of a PEO homopolymer by SANS reveal clearly reduced values compared to $A_{2}$ in pure water but still good solvent conditions for $\mathrm{PEO}$ in all water/DMF mixtures. However, a significant reduction in the radius of gyration was not found. Therefore, it was concluded that the reduced solvent quality has a more pronounced effect for the PEO chain dimensions in the confined geometry of a micellar corona.
\end{abstract}

\section{Introduction}

When amphiphilic block copolymers are dissolved in a selective solvent, i.e., a solvent which is good for one block but a precipitant for the other, they spontaneously self-assemble into supramolecular aggregates known as micelles. The micelles often display a spherical geometry in which the insoluble block forms the inner part or core, whereas the soluble block forms a solvent-rich shell or corona. The general behavior of block copolymers in selective solvents has been subject of copious theoretical and experimental studies during the past decades. They are reviewed in several books ${ }^{1,2}$ and review articles ${ }^{3-8}$ related to this topic.

Generally, the micellization process is driven by the systems tendency to lower the interfacial free energy by minimizing the area of the insoluble block exposed to the solvent. According to scaling theories, micellar growth, on the other hand, is mainly counterbalanced by chain stretching of the core and corona chains which increases the free energy of micellization. The relative contributions of core and corona chain stretching to the free energy depend on the core and shell dimensions primarily determined by block copolymer molar mass characteristics. In this context two limiting structural cases are considered, i.e., starlike and crew cut micelle, ${ }^{8}$ in which contributions from either core or corona chain deformation are neglected. For tuning micellization properties of an individual block copolymer, with given molar mass and composition, modification of the interfacial free energy is necessary. This can be achieved by using mixed solvents of different quality for the coreforming block, ${ }^{9}$ thereby adjusting the interfacial tension, $\gamma$, between solvent and polymer.

The effect of interfacial tension on micellization of polystyrene-poly(ethylene oxide) (PS-PEO) block co- polymers in water and tetrahydrofuran (THF) as cosolvent has been recently studied by Seo et al. ${ }^{10}$ using laser light scattering and contact angle measurements. Since THF is a good solvent also for polystyrene, it acts as a plasticizer and prevents the core to be in a glassy state. Therefore, this system was assumed to be in thermodynamic equilibrium. However, because of nonuniform partition of THF in core, shell, and solvent interpretation of data becomes complicated, and therefore, this system cannot be considered as a good model system to study the general influence of interfacial tension on the micellization behavior of block copolymers.

The aim of the present study is to scrutinize in more detail the role of interfacial tension for the micellization properties using a poly(ethylene-co-propylene)-poly(ethylene oxide) (PEP-PEO) block copolymer and water/ $N, N$-dimethylformamide (DMF) solvent mixtures as the model system. The molar characteristics of PEP-PEO block copolymers can be controlled by modern anionic polymerization techniques, yielding low polydispersities and predefined molar masses and compositions. ${ }^{11}$ In contrast to PS, the PEP block has a low glass transition temperature of $-56{ }^{\circ} \mathrm{C}$ and is incompatible with both water and DMF. Previous small-angle neutron scattering studies have shown that PEP-PEO block copolymers form well-defined micelles in water with segregated core-shell structures and large aggregation numbers. ${ }^{12}$ This is essentially due to the high incompatibility of PEP and water which is expressed in a large interfacial tension between these two components. The influence of block copolymer molar mass and composition has been studied previously. ${ }^{13-15}$ Different morphologies ranging from long cylinders to spherical micelles either with starlike or homogeneous density profile in the corona were observed. In parallel per- 
formed kinetic studies have shown that the micelles are kinetically frozen since unimer exchange is practically not existent in the $\mathrm{PEP}-\mathrm{PEO} /$ water system. Unimer exchange was observed in DMF, which is less incompatible with PEP. ${ }^{16}$ This is reflected by a considerable reduction in $\gamma$ leading to an acceleration of unimer exchange and at the same time to a strong decrease in the aggregation number of the micelles.

In this work we will focus on the global aggregation behavior of a highly asymmetric PEP-PEO block copolymer in water/DMF mixtures by small-angle neutron scattering and pendant drop tensiometry. We have investigated a fully deuterated and a fully protonated block copolymer with almost the same degree of polymerization designated as d-PEP1-d-PEO20 and h-PEP1h-PEO20, respectively, where the numbers indicate the nominal molar mass of the different blocks, as a function of DMF mole fraction in the solvent mixture. The large asymmetry in the block composition was taken in order to obtain a starlike structure of the micelles. The chosen molar mass ensures that the size of the micelles can be well resolved by small-angle neutron scattering. The two oppositely labeled block copolymers were taken since this type of labeling is essential for the investigation of chain exchange kinetics, which were performed in parallel. The SANS pattern were analyzed by a spherical core-shell model which has been shown to be a suitable model for the structural characterization of this type of micelles. ${ }^{12,13}$ The interfacial tension was determined from a PEP homopolymer in the respective solvent mixtures. The quality of the solvent with respect to PEO was measured in terms of the second virial coefficient, $A_{2}$, by SANS on a PEO homopolymer in the dilute concentration range. The obtained results are discussed in the framework of different thermodynamical models.

\section{Theoretical Background}

A number of theoretical models for the micellization behavior of polymeric micelles have been proposed. They can coarsely be divided into "mean field"17-19 and "scaling"20-22 theories. All theories commonly rely on the pseudophase approximation, i.e., the assumption that the micelles can be viewed as a separate thermodynamic phase. This applies well above the critical micelle concentration, $c \gg \mathrm{cmc}$, where unimer concentration is low and, in addition, aggregation numbers are large, $P$ $\gg 1$, and narrowly distributed. In all models the free energy associated with the interfacial tension is considered to be the driving force for micellization. They essentially differ in the way the counteracting free energy is calculated, in particular the free energy of the corona.

Mean field theories commonly neglect the effects of excluded volume on the corona structure and assume a constant density. A substantial mean field theory for spherical micelles with segregated cores was developed by Nagarajan and Ganesh. ${ }^{19}$ They numerically calculated the free energy of a single micelle, $F_{\text {micelle, }}$, starting from a single dispersed block copolymer in infinite dilution as a reference state. Six contributions to the free energy of micellization are considered: two terms accounting for the free energy of deformation due to stretching of the two blocks, two terms describing the free energy change associated with the energy of mixing, one term from the entropy of localization, and one from the free energy associated with the formation of an interface. The results are generally system specific; however, combining the numerical results of five different systems, they were able to establish the following general relation for the aggregation number:

$$
\begin{aligned}
& P_{\text {mean field }}= \\
& \frac{4 \pi m_{\mathrm{B}}\left(\gamma l^{2} / k_{\mathrm{b}} T\right)+(4 \pi / 3) m_{\mathrm{B}}{ }^{1 / 2}+(4 \pi / 3) m_{\mathrm{A}}{ }^{1 / 2}\left(R_{\mathrm{c}} / D\right)}{1+m_{\mathrm{B}}{ }^{-1 / 3}+m_{\mathrm{B}} / m_{\mathrm{A}}\left(D / R_{\mathrm{c}}\right)^{2}}
\end{aligned}
$$

where $m_{\mathrm{A}}$ and $m_{\mathrm{B}}$ are effective number of repeating units of the soluble and the insoluble block, respectively, $l$ is a characteristic segment length, $D$ is the corona or brush size, and $R_{\mathrm{m}}$ is the overall micellar radius. $k_{\mathrm{b}}$ denotes the Boltzmann constant and $T$ the absolute temperature. It is important to note that according to this expression the aggregation number varies linearly with the interfacial tension.

In the scaling theories swelling of the chains is considered, allowing for a radial density profile in the corona region. Three main contributions to the micellar free energy are commonly considered:

$$
F_{\text {micelle }}=F_{\text {interface }}+F_{\text {core }}+F_{\text {corona }}
$$

$F_{\text {interface }}$ accounts for the interfacial free energy per chain between core and solvent and is taken as the product of interface area and interfacial tension $\gamma$ :

$$
F_{\text {interface }}=4 \pi R_{\mathrm{c}}^{2} \gamma / P
$$

$R_{\mathrm{c}}$ is the micellar core radius. The free energy of the core, $F_{\text {core }}$, results from the stretching of the core chains. Since the block copolymers in this study have a large asymmetry, we expect the case of starlike density profile to apply. For the starlike micelles where $N_{\mathrm{B}} \ll N_{\mathrm{A}}$ the contribution of $F_{\text {core }}$ to $F_{\text {micelle }}$ is small and will be neglected.

For the corona of a starlike micelle the free energy contribution, $F_{\text {corona }}$, is calculated following de Gennes "blob recipe". ${ }^{23}$ In this approach each blob (region of nonoverlapping chains) is assigned a thermal energy $k_{\mathrm{b}} T$. The free energy of the corona is then simply given by $F_{\text {corona }}=n_{\text {blob }} k_{\mathrm{b}} T$, with $n_{\text {blob }}$ the number of blobs in the corona. $n_{\text {blob }}$ obviously depends on the density profile of the corona. Starting from the model of Daoud and Cotton $^{24}$ for regular star polymers, which assumes a radius-dependent blob size, Halperin ${ }^{20}$ considered an effective density profile, $n(r) \sim P^{2 / 3} r^{-4 / 3}$, in the corona. This assumption should be valid for micelles formed by asymmetric block copolymers with a short insoluble and a long soluble block in a highly selective solvent.

An additional model was derived by Izzo et al. ${ }^{22}$ for the intermediate micellar regime $\left(N_{\mathrm{A}}{ }^{15 / 11} \ll N_{\mathrm{B}} \ll\right.$ $\left.N_{\mathrm{A}}{ }^{18 / 11}\right)$. This model is based on the work of de Gennes ${ }^{26}$ and Alexander ${ }^{25}$ on planar brushes. They are characterized by a parabolic density profile with a constant blob size throughout the layer.

The corresponding free energies of the coronas are given by

$F_{\text {corona }} / k_{\mathrm{b}} T \sim \begin{cases}P^{1 / 2} \ln \left(\left(D+R_{\mathrm{c}}\right) / R_{\mathrm{c}}\right) & \text { starlike } \\ P^{5 / 18} N_{\mathrm{B}}^{-5 / 9} N_{\mathrm{A}} & \text { intermediate }\end{cases}$

Minimization of $F_{\text {micelle }}$ (eq 2) using eq 4 for $F_{\text {corona }}$ and assuming $F_{\text {core }} \approx 0$ results in the following scaling 
relations for the aggregation number:

$$
P_{\text {scaling }} \sim \begin{cases}\gamma^{6 / 5} N_{\mathrm{B}}^{4 / 5} & \text { starlike } \\ \gamma^{18 / 11} N_{\mathrm{B}}{ }^{2} N_{\mathrm{A}}^{-18 / 11} & \text { intermediate }\end{cases}
$$

We emphasize the strong difference in the dependence of $P$ on $\gamma$ for the two different scaling regimes.

Furthermore, the theory of starlike micelles predicts that the corona thickness, $D$, scales like

$$
D \sim P^{1 / 5} N_{\mathrm{A}}^{3 / 5}
$$

The quantities $P$ and $D$ are determined by SANS in this study. Their dependence on $\gamma$ will be discussed within the different theoretical approaches.

\section{Experimental Section}

3.1. Synthesis and Characterization of Polymers. The polymers in this study were prepared by anionic polymerization except the PEO homopolymer, h-PEO21, which was purchased from Polymer Standard Service, Mainz, Germany. The syntheses of the two block copolymers, h-PEP1-h-PEO20 and d-PEP1-d-PEO20, were accomplished by a two-step process since the preparation of 1,4-polyisoprene, the parent material of PEP, requires reaction conditions different from those necessary for the anionic polymerization of ethylene oxide. The first step involves the polymerization of isoprene$\mathrm{d}_{8}$ (Cambridge Isotope Laboratories, Andover, MA, 98\% D) and isoprene- $\mathrm{h}_{8}$, respectively, with tert-butyllithium as initiator and benzene as solvent. The living polymers were end-capped by the addition of an excess amount of either EO-d $\mathrm{d}_{4}$ (CDNIsotopes, Quebec, Canada, 99.8\% D) or EO-h $\mathrm{h}_{4}$ and were terminated with acetic acid to obtain the fully deuterated or fully protonated precursor polymers d-PI1-OH and h-PI1-OH. The hydroxy-terminated polyisoprenes were subsequently saturated with deuterium or hydrogen by means of a conventional $\mathrm{Pd} / \mathrm{BaSO}_{4}$ catalyst, resulting in the corresponding PEP1-OH polymers. In the second step of the synthesis the PEP-OH polymers were converted into the macroinitiators, d-PEP1-OK and h-PEP1-OK, by titration with naphthalene potassium in THF. The macroinitiators were used to polymerize deuterated or protonated ethylene oxide in THF at $50{ }^{\circ} \mathrm{C}$ for 2 days. After termination with a small amount of acetic acid the block copolymers were precipitated at $-20^{\circ} \mathrm{C}$ in acetone and finally freeze-dried from benzene. A more detailed description of the synthesis of PEP-PEO block copolymers has been published earlier. ${ }^{11}$ An analogous h-PEP1 homopolymer was synthesized under the same conditions as described before for the preparation of h-PEP1-OH. Instead of adding ethylene oxide, the living polymer was terminated with degassed methanol.

The PI precursor polymers were examined by size exclusion chromatography (SEC) with a Waters $150 \mathrm{C}$ instrument at 30 ${ }^{\circ} \mathrm{C}$. Five styragel columns covering a nominal porosity range of $100-10^{5} \AA$ and tetrahydrofuran as eluant at a flux rate of $1 \mathrm{~mL} / \mathrm{min}$ were used in combination with the Waters $150 \mathrm{C}$ internal refractive index detector. The system was conventionally calibrated with a set of narrow molar mass polystyrene standards. For the three PI homopolymers, polydispersities of 1.07 were obtained. A comparison of the elution volumes of $\mathrm{d}-$ and h-PI1-OH revealed a slightly higher degree of polymerization for the deuterated homopolymer, $N_{\mathrm{d}-\mathrm{PEP}} / N_{\mathrm{h}-\mathrm{PEP}}=$ 16/15. After saturation the corresponding PEP homopolymers were remeasured by SEC. Detectable changes in polydispersity could not be observed in any case.

Absolute molar masses of the two protonated polyisoprenes were determined by ${ }^{1} \mathrm{H}$ NMR measurements in deuteriochloroform. The spectra were recorded on a Bruker AMX 300 spectrometer. The signal of the nine protons of the initiator group was taken as internal reference for the calculation of the number-average molar mass, $M_{\mathrm{n}} .{ }^{1} \mathrm{H}$ NMR spectra were also recorded from the PEP samples, including the deuterated
Table 1. Molar Mass Characteristics of the Polymers

\begin{tabular}{lcccccccc}
\hline & \multicolumn{3}{c}{ PEP } & & \multicolumn{3}{c}{ PEO } \\
\cline { 2 - 4 } \cline { 6 - 8 } \multicolumn{1}{c}{ polymer } & $M_{\mathrm{n}}$ & $M_{\mathrm{w}} / M_{\mathrm{n}}$ & $N_{\mathrm{PEP}}$ & & $M_{\mathrm{n}}$ & $M_{\mathrm{w}} / M_{\mathrm{n}}$ & $N_{\mathrm{PEO}}$ \\
\hline h-PEP1-h-PEO20 & 1100 & 1.06 & 15 & & 21900 & $1.04^{a}$ & 497 \\
d-PEP1-d-PEO20 & 1400 & 1.06 & 16 & & 23900 & $1.04^{a}$ & 497 \\
h-PEP1 & 1300 & 1.07 & 18 & & & & \\
h-PEO21 & & & & $21200^{b}$ & 1.06 & 481
\end{tabular}

${ }^{a}$ Overall polydispersity of the PEP-PEO block copolymer. ${ }^{b}$ Nominal molar mass.

material. The absence of signals arising from olefinic protons confirmed complete hydrogenation of the materials. The molar mass of the deuterated polyisoprene was obtained from titration of the d-PEP1-OH with naphthalene potassium. The result confirms that the degree of polymerization is slightly higher than that one of h-PEP1-OH as already found by SEC for the unsaturated PI polymers.

SEC measurements of the two PEP-PEO block copolymers were recorded with the same chromatography setup but with $\mathrm{THF} / N, N$-dimethylacetamide (90:10 by volume) as eluant in order to suppress interaction of the polar $\mathrm{PEO}$ with the column material. The SEC traces reveal narrow molar mass distributions with polydispersities smaller than 1.04 as determined by PS calibration. The elution volumes of both block copolymers are almost identical, indicating the same degree of polymerization. The SEC traces further reveal the absence of PEP homopolymer in both cases. PEO homopolymer, on the other hand, cannot be excluded from SEC analysis, since the elution volumes of the polymers are nearly the same. However, in analogy to the synthesis of symmetric PEP-PEO block copolymers, in which the absence of homopolymer was satisfactorily shown, ${ }^{11}$ we assume also for the two asymmetric samples pure block copolymers without any homopolymer contamination.

The h-PEP1-h-PEO20 block copolymer was additionally characterized by ${ }^{1} \mathrm{H}$ NMR. On the basis of the known molar mass of h-PEP1-OH, the degree of polymerization of the h-PEO20-block was calculated by relating the intensity of the PEO methylene signal at $3.5 \mathrm{ppm}$ with the intensities from PEP between 0.6 and $1.3 \mathrm{ppm}$. The degree of polymerization of the d-PEO20-block in the fully deuterated block copolymer was assumed to be the same as determined for the h-PEO20block since elution volumes of the two block copolymers were identical. The molar characteristics of all polymers are depicted in Table 1.

3.2. Density and Interfacial Tension. To determine interfacial tensions with high accuracy, precise values for the mass density of each individual component have to be known. Densities were measured with an oscillation U-tube density meter using the Anton Paar DMA 5000 instrument. The theoretical accuracy of this instrument is specified to be $1 \times$ $10^{-5} \mathrm{~g} / \mathrm{cm}^{3}$. Water purified by a Millipore apparatus was taken for all measurements. Its density, $d$, was determined to be $0.99816 \mathrm{~g} / \mathrm{cm}^{3}$ at $20{ }^{\circ} \mathrm{C}$. The almost exact agreement with the tabulated density of water at this temperature $(d=0.99820$ $\mathrm{g} / \mathrm{cm}^{3}$ ) served as an additional validity check for the quality of the used $\mathrm{H}_{2} \mathrm{O} . \mathrm{D}_{2} \mathrm{O}$ (Aldrich, 99.8\% D), DMF (Fluka, p.A.

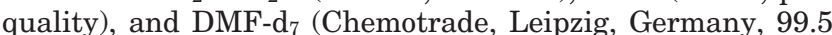
D) were used as received without further purifications. The density of $\mathrm{D}_{2} \mathrm{O}$ at $20{ }^{\circ} \mathrm{C}$ was measured to be $1.10525 \mathrm{~g} / \mathrm{cm}^{3}$, and those of DMF and DMF- $\mathrm{d}_{7}$ were 0.94858 and 1.04467 $\mathrm{g} / \mathrm{cm}^{3}$, respectively. The DMA 5000 instrument was also used to measure the bulk densities of h-PEP1, h-PEP1-OH, and d-PEP1-OH. This was possible since the low viscosities of these materials allow to fill the U-tube of the density meter properly. The measured density at $20{ }^{\circ} \mathrm{C}$ was $0.84042 \mathrm{~g} / \mathrm{cm}^{3}$ for h- PEP1 and $0.85299 \mathrm{~g} / \mathrm{cm}^{3}$ for h-PEP1-OH. For the deuterated material the density was determined to be $0.97067 \mathrm{~g} / \mathrm{cm}^{3}$. In the temperature range between 20 and $60{ }^{\circ} \mathrm{C}$ the densities of the three PEP samples show a simple linear relationship according to $d(T)=A+B T$, with $A=0.86510 \mathrm{~g} / \mathrm{cm}^{3}, B=-6.0515 \times$ $10^{-4} \mathrm{~g} /\left(\mathrm{cm}^{3} \mathrm{~K}\right)$ for h-PEP1-OH, $A=0.98461 \mathrm{~g} / \mathrm{cm}^{3}, B=-6.9629$ $\times 10^{-4} \mathrm{~g} /\left(\mathrm{cm}^{3} \mathrm{~K}\right)$ for d-PEP1-OH, and $A=0.85246 \mathrm{~g} / \mathrm{cm}^{3}, B=$ $-6.0220 \times 10^{-4} \mathrm{~g} /\left(\mathrm{cm}^{3} \mathrm{~K}\right)$ for $\mathrm{h}-\mathrm{PEP} 1$. In addition to the pure 
Table 2. Densities of Water/DMF Mixtures and Interfacial Tensions of h-PEP1 in the Same Mixtures

\begin{tabular}{lccc}
\hline$X_{\mathrm{DMF}}$ & $T\left[{ }^{\circ} \mathrm{C}\right]$ & $d\left[\mathrm{~g} \mathrm{~cm}^{-3}\right]$ & $\gamma\left[\mathrm{mN} \mathrm{m}^{-1}\right]$ \\
\hline 0 & 20 & 0.99816 & $46.0 \pm 1.3$ \\
0.0125 & 20 & 0.99778 & $36.5 \pm 1.0$ \\
0.025 & 20 & 0.99779 & $36.0 \pm 1.0$ \\
0.05 & 20 & 0.99829 & $32.4 \pm 0.9$ \\
0.10 & 20 & 0.99972 & $27.4 \pm 0.8$ \\
0.15 & 20 & 1.00003 & $26.6 \pm 0.7$ \\
0.20 & 20 & 0.99972 & $22.0 \pm 0.6$ \\
0.25 & 20 & 0.99802 & $21.8 \pm 0.6$ \\
0.30 & 20 & 0.99545 & $19.7 \pm 0.5$ \\
0.30 & 42 & 0.97592 & $18.7 \pm 0.5$ \\
0.30 & 50 & 0.96857 & $18.0 \pm 0.4$ \\
0.35 & 30 & 0.98309 & $17.5 \pm 0.4$ \\
0.40 & 30 & 0.97932 & $16.5 \pm 0.3$ \\
0.50 & 30 & 0.97546 & $14.5 \pm 0.3$ \\
0.60 & 30 & 0.96512 & $12.3 \pm 0.3$ \\
0.65 & 30 & 0.95982 & $11.6 \pm 0.3$ \\
0.70 & 30 & 0.95841 & $11.3 \pm 0.3$ \\
1.0 & 20 & 0.94858 & $8.6 \pm 0.2$
\end{tabular}

components, the densities of the solvent mixtures were determined. While isotopic mixtures of $\mathrm{H}_{2} \mathrm{O}, \mathrm{D}_{2} \mathrm{O}$, and $\mathrm{DMF}, \mathrm{DMF}$ $\mathrm{d}_{7}$ behave ideally, mixtures of water and DMF were found to be nonideal, showing nonadditivity of molar volumes. Therefore, the density of each solvent mixture was measured separately at the experimental temperatures. Values are shown in Table 2.

Determination of the interfacial tension was performed with the axisymmetric drop shape analysis technique using a commercial pendant drop tensiometer of Krüss, Germany, model G10, equipped with DSA1 software. Images of the axisymmetric drops were obtained and digitalized by a frame grabber. An external thermostat was coupled to the sample chamber, allowing the temperature to be kept within $\pm 0.1^{\circ} \mathrm{C}$ from the target temperature. Measurements were performed at the same temperatures as for the SANS and density measurements. Prior to the measurements, sample container, needle, and syringe were thoroughly cleaned and dried since interfacial tensions are very sensitive to impurities. Only protonated solvents were used for the determination of $\gamma$. To maintain a high degree of comparison, the solvents were identical with those used in the isotopic mixtures for density and SANS measurements. The quality of $\mathrm{H}_{2} \mathrm{O}$ and $\mathrm{D}_{2} \mathrm{O}$ was additionally checked by surface tension measurements at the liquid-air interface. For both $72.5 \pm 0.1 \mathrm{mN} / \mathrm{m}$ at $20{ }^{\circ} \mathrm{C}$ was obtained. The good agreement with reported literature values $(\gamma=72.3-72.8 \mathrm{mN} / \mathrm{m})$ again indicates high purity. For measuring $\gamma$ the solvent or solvent mixture was filled into a quartz glass container and then placed into the sample chamber. A drop of the h-PEP1 polymer was prepared in the solvent using a syringe with a curved needle since due to the lower density of PEP the drop stays in the upright position. Drop images were taken and analyzed every $5 \mathrm{~s}$. After $2-3 \mathrm{~h}$ $\gamma$ reached an asymptotic plateau value. The average value of at least two measurements was taken as interfacial tension. Values are listed in Table 2. Additionally, measurements of $\mathrm{h}-\mathrm{PEP} 1-\mathrm{OH}$ and d-PEP1-OH were performed in $\mathrm{D}_{2} \mathrm{O}$. Within experimental error for both polymers the same interfacial tension $\gamma=29.9 \pm 0.1 \mathrm{mN} / \mathrm{m}$ was found. The interfacial tension of d-PEP1-OH could not be determined in $\mathrm{H}_{2} \mathrm{O}$ since the densities of both components are almost equal.

3.3. SANS Experiments and Data Evaluation. The SANS measurements were performed on the KWS2 instrument at the research reactor FRJ2 at Forschungszentrum Jülich $\mathrm{GmbH}$, Germany. The SANS samples were prepared by swelling the polymer in the respective solvent mixture at room temperature first and then by heating to $65-70{ }^{\circ} \mathrm{C}$ until any sign of turbidity vanished. After cooling to room temperature the samples were subsequently stirred for at least 1 day at the measuring temperature. Finally, optically clear and slightly viscous solutions were obtained. In samples containing more than $30 \mathrm{~mol} \%$ DMF precipitation of the polymer occurred at room temperature. After warming slightly the polymer could be redissolved easily. All measurements on these samples were therefore performed at $30{ }^{\circ} \mathrm{C}$. Most of the protonated block copolymer micelles were studied at polymer volume fractions of $\phi \approx 0.25 \%$ in fully deuterated solvent, providing full contrast. Some of the protonated micelles and almost all of the deuterated micelles were studied at reduced contrast in an isotopic solvent mixture $(50-60 \%$ protonated solvent) because this investigation was part of a kinetic study, where zero average contrast conditions were applied. Several samples were double checked in both contrasts. Within experimental uncertainty the same results were obtained.

The PEO homopolymer, h-PEO21, was studied in full contrast, i.e., in deuterated solvents. Samples with concentrations between 1.2 and 0.1 vol \% were prepared using the same procedure as for the micelles. Prior to sample preparation the solvent mixtures were filtrated through $20 \mathrm{~nm}$ membrane filters (Anotop, Whatman, UK). The solutions were filtrated twice through $200 \mathrm{~nm}$ Anotop membrane filters. This procedure was performed in order to avoid possible PEO selfaggregation due to impurities and dust. ${ }^{33}$ The overlap concentration of h-PEO21 was estimated from the intrinsic viscosity, $\phi^{*} \simeq 1 /[\eta]$, to be $\approx 3$ vol $\%$. [ $\left.\eta\right]$ was calculated from the MarkHouwink-Sakurada relationship derived from data reported by Woodley et al. ${ }^{34}$

Solutions containing deuterated solvents were investigated in $2 \mathrm{~mm}$ Hellma quartz cells, and those containing pure protonated solvents were studied in $1 \mathrm{~mm}$ cells to avoid low transmission due to incoherent scattering. Transmission values were typically between $50 \%$ and $80 \%$ depending on the relative hydrogen content of the solvent.

The measurements were performed at three instrument setups with sample-to-detector distances of $L=2,8$, and 20 $\mathrm{m}$. The collimation was chosen to be equal to $L$ except at $2 \mathrm{~m}$, where $4 \mathrm{~m}$ collimation was taken in order to avoid dead time effects and also to improve the resolution. The neutron wavelength was $\lambda=7.2 \AA$ with a spread of $\Delta \lambda / \lambda \approx 10 \%$. With these setups the scattering vector $Q$ covers a range of $2 \times 10^{-3}$ $\AA^{-1} \leq Q \leq 0.11 \AA^{-1}(Q=4 \pi \sin (\theta / 2) / \lambda$, where $\theta$ is the scattering angle).

The raw data were corrected two-dimensionally for the empty cell scattering. Detector sensitivity corrections were made with Lupolen, which also served as a secondary standard for absolute calibration according to

$$
\frac{\mathrm{d} \Sigma}{\mathrm{d} \Omega_{\mathrm{s}}}(Q)=\frac{T_{\mathrm{l}} d_{\mathrm{l}}(\mathrm{d} \Sigma / \mathrm{d} \Omega)_{\mathrm{l}} L_{\mathrm{s}}^{2}}{T_{\mathrm{s}} T_{\mathrm{ec}} d_{\mathrm{s}} I_{\mathrm{l}} L_{\mathrm{l}}^{2}}\left(I_{\mathrm{s}}(Q)-T_{\mathrm{s}} I_{\mathrm{ec}}(Q)\right)
$$

where subscripts $1, \mathrm{~s}$, and ec denote Lupolen, sample, and empty cell, respectively. $T$ is the transmission, $I$ the intensity, and $d$ the sample thickness. The measured intensities were normalized to the same monitor count rate in order to take into account a timely varying neutron flux. $I_{1}$ was replaced by a detector average since Lupolen is a flat incoherent scatterer. $(\mathrm{d} \Sigma / \mathrm{d} \Omega)_{1}$ of Lupolen has been determined by calibration against vanadium to be $1.8155 \mathrm{~cm}^{-1}$. After radial averaging, the calculated incoherent contribution of the polymer as well as the measured solvent scattering was subtracted to obtain the coherent macroscopic scattering cross section, $(\mathrm{d} \Sigma / \mathrm{d} \Omega)(Q)$, of the particle.

Several models have been proposed in the past to describe small-angle scattering data of block copolymer micelles. An overview on the different approaches has recently been summarized in two review articles. ${ }^{35,36}$ The most advanced and comprehensive model has been presented by Svaneborg and Pedersen, ${ }^{37-39}$ explicitly taking into account the short-range correlations and excluded-volume interactions of the corona chains. In this work we use an alternative model which has been successfully applied in previous studies on PEP-PEO micelles in water. ${ }^{12,13} \mathrm{~A}$ similar approach has been used by Won et al..$^{40}$ to characterize polybutadiene-PEO micelles in aqueous solution.

Generally, the coherent macroscopic scattering cross section measured in a SANS experiment has the following form: 


$$
\frac{\mathrm{d} \Sigma}{\mathrm{d} \Omega}(Q)=n_{z}\left\langle|A(Q)|^{2}\right\rangle
$$

where $n_{z}$ denotes the number density of scatterers and $A(Q)$ the scattering amplitude. Assuming that the critical micelle concentration is low $n_{z}$ can be approximated by

$$
n_{z}=\frac{\phi}{P\left(V_{\mathrm{PEP}}+V_{\mathrm{PEO}}\right)}
$$

with $V_{\mathrm{PEP}}$ and $V_{\mathrm{PEO}}$ as the volumes of the blocks of a single polymer chain. For a micellar structure consisting of a segregated PEP core and a PEO corona we can model the scattering amplitude by

$$
A(Q)=P V_{\mathrm{PEP}}\left(\rho_{\mathrm{PEP}}-\bar{\rho}_{0}\right) A(Q)_{\mathrm{c}}+P V_{\mathrm{PEO}}\left(\rho_{\mathrm{PEO}}-\bar{\rho}_{0}\right) A(Q)_{\mathrm{sh}}
$$

Here $\bar{\rho}_{0}$ denotes the scattering length density of the solvent, which for the mixtures is calculated by

$$
\bar{\rho}_{0}=\frac{\sum x_{i} b_{i}}{v}
$$

with $x_{i}$ the mole fraction of component $i$ in the solvent mixture, $b_{i}$ the sum of the coherent scattering length of all atoms in a solvent molecule, and $v$ the average volume of a solvent molecule given by

$$
v=\frac{\sum x_{i} M_{i}}{d N_{\mathrm{Avo}}}
$$

$M_{i}$ is the molar mass of the solvent component $i, N_{\mathrm{Avo}}$ the Avogadro number, and $d$ the density of the solvent mixture. The scattering length densities are tabulated in Table 3. $\rho_{\mathrm{PEP}}$ and $\rho_{\text {PEO }}$ are the scattering length densities of the polymers calculated by a formula equivalent to eq 11 . $\rho$ PEP was calculated to be $-3.014 \times 10^{9}$ and $7.177 \times 10^{10} \mathrm{~cm}^{-2}$ at $20^{\circ} \mathrm{C}$ for the protonated and deuterated material, respectively. $v_{\text {PEP }}$ was determined from the measured density of PEP1-OH at $20^{\circ} \mathrm{C}$. At higher temperatures corresponding densities were used. For PEO scattering length densities of $6.356 \times 10^{9}$ and $7.024 \times$ $10^{10} \mathrm{~cm}^{-2}$ were calculated. For $v_{\text {PEO }}$ the value of amorphous PEO at $20{ }^{\circ} \mathrm{C}, d=1.125 \mathrm{~g} / \mathrm{cm}^{3}$, was taken. Temperature corrections at higher temperatures were made according to data given by Smith et al. ${ }^{41}$ For all deuterated materials the nominal degree of deuteration was considered for the calculations.

It should be noted that the apparent solution density of PEO is about $7 \%$ higher than its bulk density as measured by Vennemann et al. ${ }^{42}$ and more recently by Sommer et al. ${ }^{43}$ on aqueous PEO solutions. This is in agreement with our own density measurements. Accordingly, the scattering length density for PEO in aqueous solution has to be calculated from the solution density. However, contrast variation experiments on the PEP1-PEO20 system have shown that the scattering cross section can best be described by the scattering length density calculated on the basis of the bulk density, $d=1.125$ $\mathrm{g} / \mathrm{cm}^{3}$. Presently, there is no explanation for this discrepancy, but one may speculate about preferential hydration by $\mathrm{D}_{2} \mathrm{O}$ or $\mathrm{H}_{2} \mathrm{O}$. A conclusive understanding would need a separate thorough investigation. However, our main results, e.g., the scaling behavior of $P$ with $\gamma$, are not affected as a change in contrast mainly leads to a small difference in the absolute values of $P$. Therefore, this issue will not be discussed further in this paper.

The partial scattering amplitudes, $A(Q)_{\mathrm{c}, \mathrm{sh}}$, for core and shell in spherical symmetry can be written as the Fourier transform of the radial density distribution, $n(r):{ }^{16}$

$$
A(Q)_{\mathrm{c}, \mathrm{sh}}=\frac{1}{C} \int_{0}^{\infty} 4 \pi r^{2} n(r) \frac{\sin (Q r)}{Q r} \mathrm{~d} r
$$

In this equation $C=\int_{0}^{\infty} n(r) 4 \pi r^{2} \mathrm{~d} r$ is a normalization con-

\begin{tabular}{|c|c|c|c|c|c|c|c|}
\hline \multirow[b]{2}{*}{$X_{\mathrm{DMF}}$} & \multirow[b]{2}{*}{$\begin{array}{c}T \\
{\left[{ }^{\circ} \mathrm{C}\right]}\end{array}$} & \multicolumn{3}{|c|}{$\mathrm{hh}$} & \multicolumn{3}{|c|}{$\mathrm{dd}$} \\
\hline & & $\begin{array}{c}\bar{\rho}_{0} \\
{\left[10^{10} \mathrm{~cm}^{-2}\right]}\end{array}$ & $P$ & $\begin{array}{l}R_{\mathrm{m}} \\
{[\AA \mathrm{A}]}\end{array}$ & $\begin{array}{c}\bar{\rho}_{0} \\
{\left[10^{10} \mathrm{~cm}^{-2}\right]}\end{array}$ & $P$ & $\begin{array}{l}R_{\mathrm{m}} \\
{[\AA]}\end{array}$ \\
\hline 0 & 20 & 6.3648 & 120 & 299 & -0.5598 & 123 & 299 \\
\hline 0.0125 & 20 & 3.5086 & 88 & 282 & 3.5086 & 97 & 276 \\
\hline 0.025 & 20 & 3.4981 & 89 & 282 & 3.4981 & 90 & 274 \\
\hline 0.05 & 20 & 3.4725 & 91 & 278 & 3.4725 & 85 & 276 \\
\hline 0.10 & 20 & 3.4691 & 74 & 268 & 3.4691 & 78 & 265 \\
\hline 0.15 & 20 & 3.4207 & 60 & 242 & 3.4207 & 62 & 252 \\
\hline 0.20 & 20 & 3.4674 & 60 & 237 & 3.4674 & 64 & 248 \\
\hline 0.25 & 20 & 3.4636 & 53 & 226 & 3.4636 & 57 & 236 \\
\hline 0.30 & 20 & 6.5863 & 47 & 222 & 3.4976 & 51 & 222 \\
\hline 0.30 & 30 & 6.5552 & 48 & 217 & & & \\
\hline 0.30 & 42 & 6.4553 & 46 & 217 & & & \\
\hline 0.30 & 50 & 6.4059 & 45 & 214 & & & \\
\hline 0.35 & 30 & 6.5178 & 45 & 209 & & & \\
\hline 0.40 & 30 & 6.5052 & 50 & 202 & & & \\
\hline 0.50 & 30 & 3.5888 & 42 & 196 & 3.5888 & 46 & 213 \\
\hline 0.60 & 30 & 6.4556 & 40 & 192 & & & \\
\hline 0.65 & 30 & 6.4299 & 29 & 191 & & & \\
\hline 0.70 & 30 & 6.4202 & 24 & 189 & & & \\
\hline 1.0 & 30 & 6.3652 & 1 & $75^{a}$ & & & \\
\hline
\end{tabular}
stant.
Table 3. Scattering Length Densities of Water/DMF Mixtures and the Fit Results of the Spherical Core-Shell Model of h-PEP1-h-PEO20 (hh) and d-PEP1-d-PEO20 (dd)

${ }^{a}$ Apparent $R_{\mathrm{g}}$ as determined from a Beaucage form factor.

For the micellar core we assumed a constant density profile, $n(r)=1$. By convoluting with a Gaussian distribution function, we take into account possible surface roughness or a diffuse core-corona interface. This leads to

$$
A(Q)_{\mathrm{c}}=\frac{3\left(\sin \left(Q R_{\mathrm{c}}\right)-Q R_{\mathrm{c}} \cos \left(Q R_{\mathrm{c}}\right)\right)}{\left(Q R_{\mathrm{c}}\right)^{2}} \exp \left(-\left(\sigma_{\mathrm{c}} Q\right)^{2} / 4\right)
$$

with $\sigma_{\mathrm{c}}$ as the smearing parameter. $R_{\mathrm{c}}$ denotes the core radius given by

$$
R_{\mathrm{c}}=\left(3 P V_{\mathrm{PEP}} /(4 \pi)\right)^{1 / 3}
$$

assuming a compact solvent- and PEO-free core containing only PEP chains with volume $P V_{\text {PEP. For the corona a }}$ hyperbolic density distribution, $r^{-x}$, was taken. The density profile was convoluted with a Fermi function that serves as a cutoff function taking into account finite chain length:

$$
n(r)=\frac{r^{-x}}{1+\exp \left(\left(r-R_{\mathrm{m}}\right) /\left(\sigma_{\mathrm{m}} R_{\mathrm{m}}\right)\right)}
$$

with $R_{\mathrm{m}}$ as the overall micelle radius and $\sigma_{\mathrm{m}}$ the corresponding smearing parameter. $x$ is a general power law exponent which in the case of constant density takes the value 0 and for starlike structures $4 / 3.13$

To describe the data at high $Q$, an additional term to eq 8 is introduced taking into account short-range fluctuations of the polymer segment density in the corona. To describe this so-called "blob scattering", a function derived by Dozier et al. ${ }^{27}$ is included. This function describes chain correlations in a blob similar to those in a semidilute polymer solution:

$$
\frac{\mathrm{d} \Sigma}{\mathrm{d} \Omega_{\text {blob }}}(Q)=\left(\rho_{\mathrm{PEO}}-\bar{\rho}_{0}\right)^{2} \frac{4 \pi \beta \sin \left(\mu \tan ^{-1}(Q \xi)\right)}{Q \xi\left(1+Q^{2} \xi^{2}\right)^{u / 2}} \Gamma(\mu)
$$

where $\beta$ is a prefactor, $\Gamma$ the gamma function, and $\xi$ the average blob radius. $\mu=1 / v-1$ with the Flory exponent $v=$ 0.5 and $v=0.588$ for Gaussian and swollen chains, respectively. Since the blob scattering relates to short-range fluctuations, no interference with the scattering amplitudes from the average core and corona is expected, and $\mathrm{d} \Sigma / \mathrm{d} \Omega_{\text {blob }}(Q)$ can simply be added to eq 8 , yielding the modified total scattering cross section: 


$$
\left.\frac{\mathrm{d} \Sigma}{\mathrm{d} \Omega}(Q)=\left.n_{z}|| A(Q)\right|^{2}\right\rangle+\frac{\mathrm{d} \Sigma}{\mathrm{d} \Omega_{\text {blob }}}(Q)
$$

Finally, the theoretical scattering cross section was convoluted with a resolution function according to Pedersen et al. ${ }^{28}$ In the standard Fortran fitting routine used, simultaneous fits of data with different resolution were performed corresponding to the different collimation and detector settings.

To describe the PEO homopolymer scattering, we have to take into account polymer interactions at finite concentrations. In the dilute regime a generalized Zimm approach was used:

$$
\frac{\mathrm{d} \Sigma}{\mathrm{d} \Omega}(Q)=\phi(1-\phi) \frac{\left(\rho_{\mathrm{PEO}}-\rho_{0}\right)^{2}}{N_{\mathrm{Avo}}}\left(\frac{1}{1 /\left(V_{\mathrm{w}}(\mathrm{PEO}) P(Q)\right)+2 A_{2} \phi}\right)
$$

Here $A_{2}$ is the second virial coefficient. A form factor $P(Q)$ derived by Beaucage ${ }^{29}$ was used to describe the data. For structures with a single spatial length scale, in this case $R_{\mathrm{g}}$, the radius of gyration, the form factor takes the form

$$
P(Q)=\exp \left(-Q^{2} R_{\mathrm{g}}{ }^{2} / 3\right)+\left(d_{\mathrm{f}} / R_{\mathrm{g}}^{d_{\mathrm{f}}}\right) \Gamma\left(d_{\mathrm{f}} / 2\right)\left(\frac{\left(\operatorname{erf}\left(Q k R_{\mathrm{g}}\right) / \sqrt{ } 6\right)^{3}}{Q}\right)^{d_{\mathrm{f}}}
$$

where erf denotes the error function, $k$ is an empirical value which for polymers is 1.06 , and $d_{\mathrm{f}}$ is the fractal dimension, i.e., $1 / v=1.7$ for a swollen chain in the good solvent limit.

By fitting the data at the different concentrations simultaneously to eqs 19 and $20, V_{\mathrm{w}}(\mathrm{PEO}), R_{\mathrm{g}}, d_{\mathrm{f}}$, and $A_{2}$ could be extracted. The theoretical form factor was finally convoluted with the resolution function described above.

\section{Results and Discussion}

The coherent macroscopic scattering cross section, $(\mathrm{d} \Sigma) / \mathrm{d} \Omega)(Q)$, of four representative h-PEP1-h-PEO20 block copolymer solutions with varying DMF content are displayed in Figure 1. In this plot $(\mathrm{d} \Sigma) / \mathrm{d} \Omega)(Q)$ is normalized by the volume fraction, the contrast, and the Avogadro number, such that the value at $Q=0$ gives the molar volume of the particle defined by $V_{\mathrm{w}}=$ $P V_{\mathrm{w}}(\mathrm{PEP}-\mathrm{PEO})$. The curves of all samples, except the one in DMF- $\mathrm{d}_{7}$, show a high-intensity plateau at low $Q$ and a shape typical for spherical scatterers indicating the formation of micelles. The intensity at low $Q$ decreases with increasing DMF content, implying successively lower aggregation. The absence of pronounced minima and maxima indicates rather diffuse corona structures characteristic for micelles with a starlike density profile. This is confirmed by comparison with SANS curves measured in a previous study on PEP-PEO samples with similar composition but higher molar mass. ${ }^{16}$ In detail, the scattering curves of the micelles show the following general behavior: a Guinier regime at low $Q$, a steep decay, $Q^{-x}, x \approx 3.5-4$, in the intermediate $Q$ range, and at high $Q$ a shallow shoulder which merges into a $Q^{-1.7}$ behavior characteristic for swollen chains. In fact, the SANS curves reflect scattering from a convolution of core and shell since the block copolymers were not labeled and no specific contrast was adjusted. Because of the large fraction of PEO, the scattering at low $Q$ is dominated by the corona. At intermediate $Q$ the intensity of the corona strongly decreases, and the small PEP core starts to contribute to the overall intensity. The shallow shoulder at high $Q$ arises from the secondary maximum in the corona partial form factor.

The data of the micellar solutions were fitted by the spherical core/shell model described above. Final fits

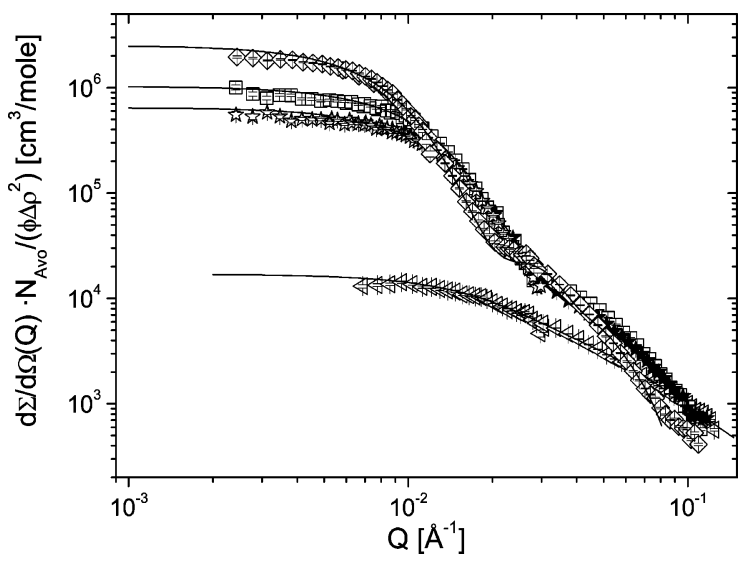

Figure 1. Coherent macroscopic scattering cross section, $(\mathrm{d} \Sigma / \mathrm{d} \Omega)(Q)$, normalized to molar volume by $N_{\mathrm{Avo}} /\left(\phi \Delta \rho^{2}\right)$ of h-PEP1-h-PEO20 block copolymer solutions at volume fractions of $\phi=0.25 \%$ in different solvents: From top to bottom: $\mathrm{D}_{2} \mathrm{O} ; \mathrm{D}_{2} \mathrm{O} / \mathrm{DMF}-\mathrm{d}_{7}, X_{\mathrm{DMF}-\mathrm{d}_{7}}=0.3 ; \mathrm{D}_{2} \mathrm{O} / \mathrm{DMF}-d_{7}, X_{\mathrm{DMF}-\mathrm{d}_{7}}=0.65 ;$ DMF- $\mathrm{d}_{7}$. Solid lines represent model fits with the spherical core-shell model or with the Beaucage form factor for the scattering curve in pure DMF- $\mathrm{d}_{7}$. For better visibility these curves are obtained by convolution of the model function with the resolution function corresponding to the intermediate $Q$ range only.

were performed with the aggregation number, $P$, the micellar radius, $R_{\mathrm{m}}$, the blob size, $\xi$, and the blob intensity parameter, $\beta$, as variables. The Flory exponent, $v$, was set to 0.588 . Block volumes, $V_{\mathrm{PEP}}$ and $V_{\mathrm{PEO}}$, concentration, $\phi$, and scattering length densities of the individual components, $\rho_{\mathrm{PEO}}, \rho_{\mathrm{PEP}}$, and $\rho_{0}$, were taken as determined or calculated. Because of the small volume fraction of the core and its small contribution to the overall intensity, the smearing parameter, $\sigma_{\mathrm{c}}$, was found to be insensitive to the fit quality. Therefore, $\sigma_{\mathrm{c}}$ was set to 0 and kept fixed for all calculations. The smearing parameter of the corona, $\sigma_{\mathrm{m}}$, was determined to be close to $10 \%$ and held constant for the overall analysis. The power law exponent $x$ of the hyperbolic density profile for the corona, $n(r) \sim r^{-x}$, was taken as a free parameter in several preliminary fits. Thereby, it was found that $(\mathrm{d} \Sigma) / \mathrm{d} \Omega)(Q)$ is perfectly fitted with $x$ $=1.3 \pm 0.1$ for all micelles. This is in agreement with results obtained earlier on PEP-PEO samples of similar asymmetry but higher molar mass. ${ }^{16}$ The value is also in agreement with the theoretical exponent of $4 / 3$ derived for regular star polymers ${ }^{24}$ and used for the case of starlike micelles. ${ }^{20}$ To reduce the number of free parameters, $x$ was fixed to $4 / 3$ in the final analysis. The resulting fits are shown as solid lines in Figure 1. In all cases a good description of the experimental data is obtained. Structure factor effects were omitted and can in fact be seen to have a negligible effect at these low concentrations of $\phi=0.25 \%$. Fitted aggregation numbers, $P$, and micellar radii, $R_{\mathrm{m}}$, are listed in Table 3 . The two parameters, $\xi$ and $\beta$, were found to be sensitive only to the high $Q$ data. Both quantities have only minor influence on the parameters $P$ and $R_{\mathrm{m}}$ describing the overall aggregation of the micelles. The blob radius can be deduced from geometrical considerations, yielding a minimum and maximum value for $\xi: \xi_{\min }=\sqrt{4 \pi R_{\mathrm{c}}^{2} / P}$; $\xi_{\max }=\sqrt{4 \pi R_{\mathrm{m}}{ }^{2} / P}$. The average blob size was estimated to be in the range of $50-70 \AA$ depending on DMF content. Good fits could be obtained using the average $\xi$ values deduced from this geometrical approach. The obtained $\beta$ values were of the order of $10^{-24} \mathrm{~cm}^{3}$, in good 


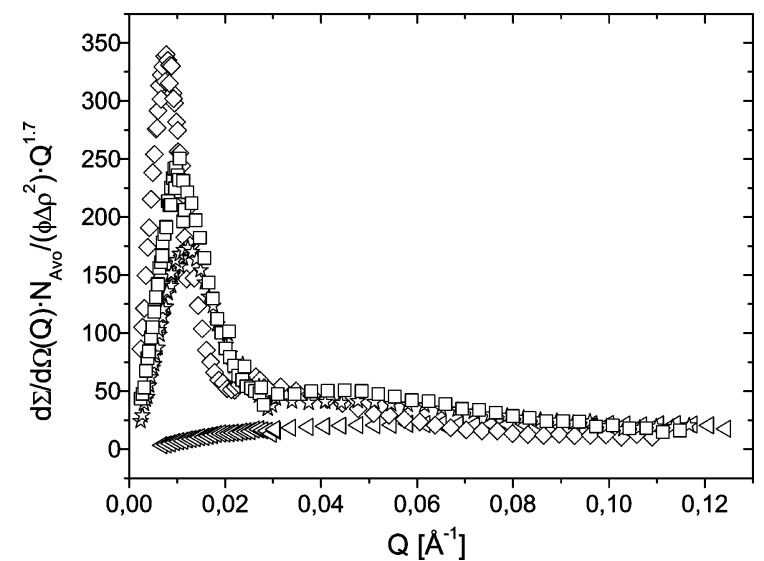

Figure 2. Generalized Kratky representation of the data in Figure 1 in the same order: From top to bottom: $\mathrm{D}_{2} \mathrm{O}$; $\mathrm{D}_{2} \mathrm{O} / \mathrm{DMF}-\mathrm{d}_{7}, X_{\mathrm{DMF}-\mathrm{d}_{7}}=0.3 ; \mathrm{D}_{2} \mathrm{O} / \mathrm{DMF}-\mathrm{d}_{7}, X_{\mathrm{DMF}-\mathrm{d}_{7}}=0.65 ;$ $\mathrm{DMF}-\mathrm{d}_{7}$.

agreement with the molar characteristics. ${ }^{31}$ However, since no contrast variation experiments on labeled polymers have been performed, these values cannot be individually determined with high accuracy. Consequently, we will leave these parameters out of discussion in the following and will focus on the global association behavior in this paper.

In pure DMF a drastic reduction of the intensity at low $Q$ and a change in the shape of the curve is observed. The shape resembles an nonaggregated linear chain in a good solvent with excluded-volume effects. That no aggregation has occurred is proved by the low intensity at $Q=0$. A conventional Zimm analysis yields for the weight-average molar volume, $V_{\mathrm{w}}=17 \times 10^{3}$ $\mathrm{cm}^{3} / \mathrm{mol}$, which agrees well with the value $V_{\mathrm{w}}=20.7 \times$ $10^{3} \mathrm{~cm}^{3} / \mathrm{mol}$ obtained from the polymer characterization. The scattering curve can be well described by the form factor of Beaucage given in eq 20 with a radius of gyration of $75 \AA$.

The scattering patterns of Figure 1 are replotted in a generalized Kratky representation, $I(Q) Q^{1 / v}$ vs $Q$, in Figure 2. In this representation a prominent feature, characteristic for branched polymers such as star polymers, is the appearance of a peak ${ }^{30}$ that disappears in the case of linear chains. This has previously been observed for regular star polymers, where the peak height is seen to decrease for decreasing arm numbers. ${ }^{32}$ This behavior is precisely observed also for the starlike PEP-PEO micelles: a strong peak in pure water that gradually decreases as the amount of DMF increases and, finally, vanishes in pure DMF for the nonaggregated block copolymer chain. At higher $Q$ values the generalized Kratky representation also reveals the weak shoulder which now becomes a maximum. At still higher $Q$ the data asymptotically merge into a plateau, suggesting the $Q^{-1.7}$ behavior of swollen coronal chains. The data further reveal a peak shift toward higher $Q$ with increasing DMF content, suggesting a decreasing size of the micelle. This feature will be discussed later.

As expected from the reduced intensity at $Q \rightarrow 0$ in Figure 1, the aggregation number, $P$, decreases as a function of the DMF content. This trend prevails for both the h-PEP1-h-PEO20 and the d-PEP1-d-PEO20 as shown in Figure 3, where $P$ is plotted vs the mole fraction DMF. However, for the latter we systematically observe a slightly higher aggregation number. This can be attributed to the slightly higher volume of the d-PEP1 compared to the h-PEP1 as determined by SEC.

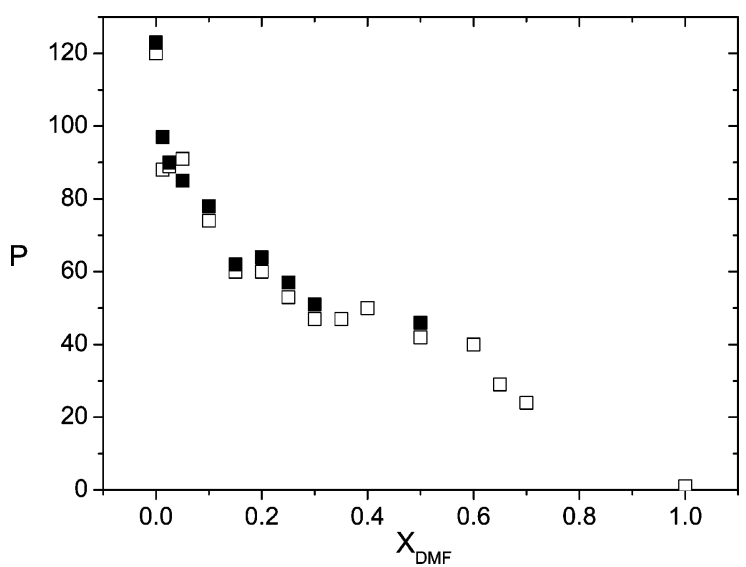

Figure 3. Aggregation number, $P$, as a function of DMF mole fraction, $X_{\mathrm{DMF}}$, in the water/DMF mixture for h-PEP1h-PEO20 (open squares) and d-PEP1-d-PEO20 (filled squares).

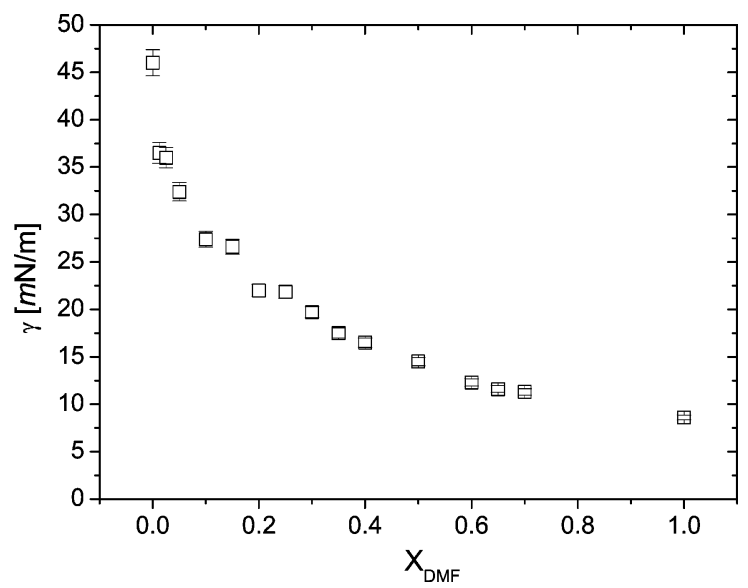

Figure 4. Interfacial tension, $\gamma$, of h-PEP1 as a function of DMF mole fraction, $X_{\mathrm{DMF}}$, in the water/DMF mixture.

As seen from the data in Table 1, we analyzed the degree of polymerization to be 16 and 15, respectively, yielding a difference in the aggregation number of $\triangle P / P$ $=5 \%$ as calculated from eq 5 for the starlike regime. Taking into account the identical values of $\gamma$ for the two precursor homopolymers in $\mathrm{D}_{2} \mathrm{O}$, it should be noted that an isotope effect is not responsible for the difference in $P$. The overall trend in aggregation reflects a decreasing incompatibility between the insoluble PEP block and the surrounding solvent as the DMF fraction increases. To elaborate and to quantify this feature in more detail, the changes in the interfacial tension were measured. This was done between a h-PEP1 homopolymer as model compound and the $\mathrm{H}_{2} \mathrm{O} / \mathrm{DMF}$ solvent mixtures. A corresponding plot is shown in Figure 4. A nearly exponential decay of the interfacial tension from $\gamma=$ $46 \mathrm{mN} / \mathrm{m}$ in pure water to $\gamma=8.6 \mathrm{mN} / \mathrm{m}$ in pure DMF is seen. Hence, DMF can be regarded as an effective tuning agent for the aggregation behavior of $\mathrm{PEP}-\mathrm{PEO}$ block copolymers in water.

In Figure 5 the aggregation number, $P$, is plotted vs $\gamma$ in a double-logarithmic representation for (a) h-PEP1h-PEO20 and (b) d-PEP1-d-PEO20. In these plots $P$ and $\gamma$ exhibit an almost linear correlation. Recalling eq 3 , the driving force for micellization is the interfacial free energy which is proportional to $\gamma$. As briefly outlined in the Theoretical Background section, the counterbalancing corona entropy differs between the theoretical approaches. Some of them predict power laws of the form $P \sim \gamma^{z}$. These are depicted in Figure 5 

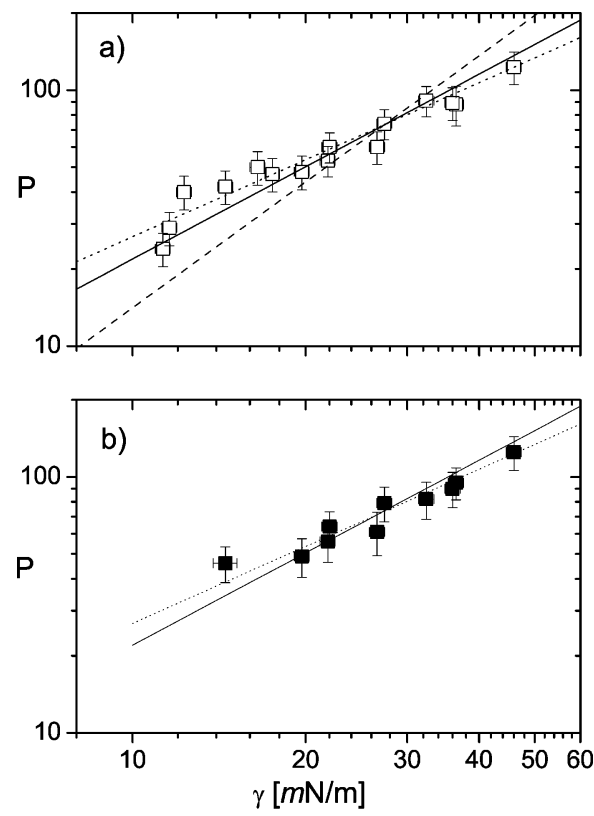

Figure 5. Interfacial tension, $\gamma$, plotted vs aggregation number, $P$, for h-PEP1-h-PEO20 in (a) and d-PEP1-d-PEO20 in (b). The lines represent different theoretical predictions: solid line: prediction of starlike micelle, $P \sim \gamma^{6 / 5}$; dashed line: prediction of intermediate micellar regime, $P \sim \gamma^{18 / 11}$; dotted line: prediction of the mean field approach, $P \sim \gamma^{1}$.

for comparison. The solid line is the prediction of the starlike model with $z=6 / 5$ (eq 5). Good agreement with the experimental results for both polymers is obtained in particular for high $P$ and $\gamma$. This is consistent with the scattering data which could be almost perfectly described by the density profile of the star model. With decreasing $P$ and $\gamma$ some systematic deviations are observed for h-PEP1-h-PEO20 shown in Figure 5a. Below $\gamma \approx 20 \mathrm{mN} / \mathrm{m}$ the aggregation numbers are above the line of the starlike prediction, before at very small $\gamma, P$ drops to 1 , representing the unaggregated block copolymer chain in pure DMF. It should be mentioned that the observed trend was confirmed by remeasuring several points in this range. Since aggregation numbers and interfacial tensions are small, the deviations may be explained by limitations of the scaling theory. Furthermore, at low $P$ the cmc is approached and the assumption of the applicability of the pseudophase approximation is generally not valid anymore.

As displayed by the pointed line, the data do not allow a distinction from the mean-field prediction of $z=1$ (eq 1). Comparing to the slope predicted by the general scaling law from the mean-field theory $(z=1)$, a good agreement is also obtained. However, in this case one would expect a constant corona density profile which is disapproved by the SANS data. Now comparing the results with the assumption of a planar brush in the intermediate regime, a much stronger dependence of the aggregation number on the interfacial tension is expected: $z=18 / 11$. The prediction is shown by the dashed line in Figure 5a and is not supported by our data. It should be noted that the variation of $P$ and $\gamma$ is less than 1 order of magnitude. Generally, such a small variation does not allow a detailed discussion within scaling theories. In tis case, however, the good agreement with the proposed scaling law, $P \sim \gamma^{6 / 5}$, for starlike micelles, and the experimentally observed density profile, $n(r) \sim r^{-4 / 3}$ consistently confirms the validity of the starlike approach and, accordingly, the existence of

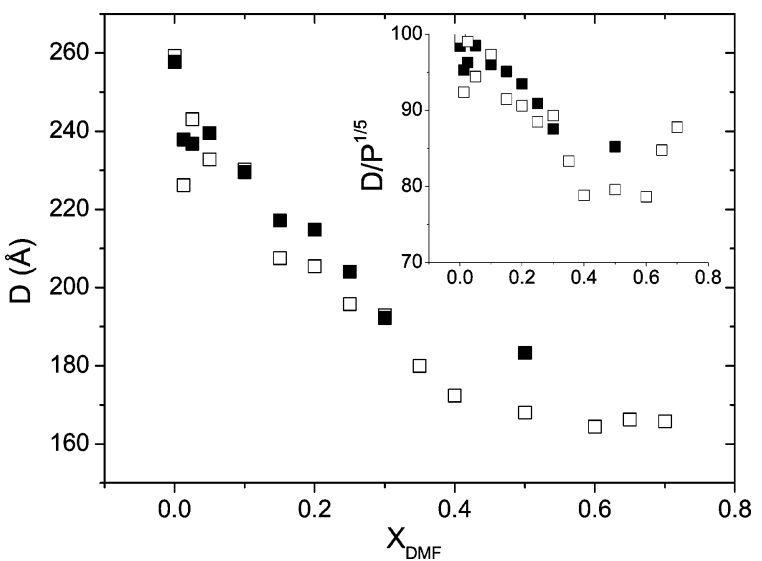

Figure 6. Brush length, $D=R_{\mathrm{m}}-R_{\mathrm{c}}$, as a function of DMF mole fraction, $X_{\mathrm{DMF}}$, in the water/DMF mixture. Inset: brush length reduced by, $P^{1 / 5}$, according to the scaling law of starlike micelles vs $X_{\mathrm{DMF}}$.

starlike micelles. For one solvent composition, $X_{\mathrm{DMF}}=$ 0.3 , the influence of temperature on the micellar properties and on the interfacial tension was studied. In the temperature range between 20 and $50{ }^{\circ} \mathrm{C}$ the aggregation number as well as $\gamma$ only slightly decreases as can be seen from the values given in Tables 2 and 3 . Changes in $R_{\mathrm{m}}$ are even less significant. Therefore, it can be concluded that the jump from 20 to $30^{\circ} \mathrm{C}$ at $X_{\mathrm{DMF}}$ $=0.3, \gamma=19.7 \mathrm{mN} / \mathrm{m}$ has only a minor influence on the discussed results.

In the following the influence of increasing DMF in the solvent mixture on the corona structure is discussed. In Figure 6 the corona thickness $D$ is plotted as a function of DMF mole fraction. $D$ was calculated by $R_{\mathrm{m}}$ $-R_{\mathrm{c}}$, where $R_{\mathrm{c}}$ was extracted from the core-shell model fit; $R_{\mathrm{c}}$ was calculated from eq 15 . An initial decrease from $259 \AA$ in pure water to about $165 \AA$ upon addition of DMF until $40 \%$ is observed. For larger fractions of DMF $D$ remains constant. To compensate for the reduction in corona size resulting from the reduction in aggregation number (eq 6 ), $D$ was scaled by $P^{-1 / 5}$. This plot is shown in the inset of Figure 6. It reveals the same trend indicating a relative shrinkage of about $20 \%$. However, for DMF fractions of $60 \%$ and $70 \% \mathrm{D} / \mathrm{P}^{1 / 5}$ appears to slightly increase again. The observed behavior was presumed to be caused by a variation of solvent quality for PEO. This supposition is supported by the experimental observation that block copolymers precipitate close to room temperature from solutions containing more than $30 \%$ DMF.

For verification we have measured the second virial coefficient, $A_{2}$, of a PEO homopolymer, h-PEO21, in three representative water/DMF solvent mixtures. The measurements were performed by SANS since static light scattering proved to be very difficult due to low contrast between PEO and DMF. As an example, the SANS results obtained in pure DMF- $\mathrm{d}_{7}$ at different volume fractions are displayed in Figure 7. The curves show at low $Q$ a high-intensity plateau and an asymptotic $Q^{-1.7}$ power law decay in the high $Q$ range. This is characteristic for polymers under good solvent conditions. The data were at first evaluated by a conventional Zimm analysis. Values obtained for $R_{\mathrm{g}}, V_{\mathrm{w}}(\mathrm{PEO})$, and $A_{2}$ are depicted in Table 4. To improve the accuracy of the fit results each data set was simultaneously fitted by the generalized Zimm approach given in eq 19, including the form factor of Beaucage (eq 20). To reduce the number of fit parameters and stabilize the fit, the 

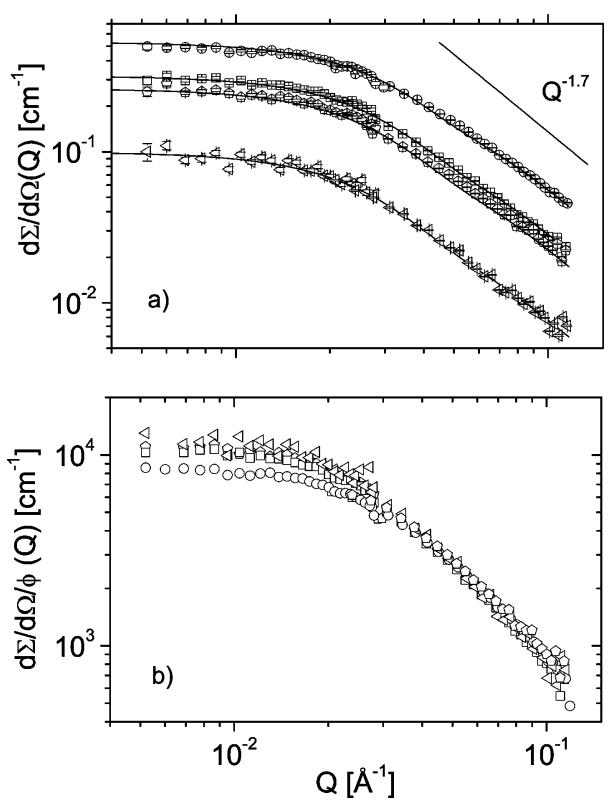

Figure 7. (a) Coherent macroscopic scattering cross section, $\mathrm{d} \Sigma(Q) / \mathrm{d} \Omega$, of h-PEO21 in pure DMF- $\mathrm{d}_{7}$ at four different concentrations: From top to bottom: $\phi=1.02 \% ; \phi=0.508 \%$; $\phi=0.4 \% ; \phi=0.13 \%$. Solid lines represent a simultaneous fit of the generalized Zimm approach given in eq 19. For better visibility these curves are obtained by convolution of the model function with the resolution function corresponding to the low $Q$ range only. (b) Same curves normalized to volume fraction.

Table 4. Results of SANS Data Evaluation of h-PEO21 in Different DMF/Water Mixtures

\begin{tabular}{lccccccc}
\hline & \multicolumn{3}{c}{ Zimm } & & \multicolumn{3}{c}{ Beaucage } \\
\cline { 2 - 3 } & $\begin{array}{c}M_{\mathrm{w}} \\
{\left[\mathrm{g} \mathrm{mol}^{-1}\right]}\end{array}$ & $\begin{array}{c}R_{\mathrm{g}} \\
{[\AA]}\end{array}$ & $\begin{array}{c}A_{2} \\
{\left[\mathrm{~cm}^{3} \mathrm{~mol} \mathrm{~g}^{-2}\right]}\end{array}$ & & $\begin{array}{c}R_{\mathrm{g}} \\
{[\AA]}\end{array}$ & $\begin{array}{c}A_{2} \\
{\left[\mathrm{~cm}^{3} \mathrm{~mol} \mathrm{~g}^{-2}\right]}\end{array}$ & $d_{\mathrm{f}}$ \\
\hline 0.30 & 16000 & 67 & $1.6 \times 10^{-3}$ & & 69 & $1.3 \times 10^{-3}$ & $1.7 \pm 0.1$ \\
0.70 & 17600 & 69 & $1.1 \times 10^{-3}$ & & 64 & $0.6 \times 10^{-3}$ & $1.8 \pm 0.1$ \\
1.0 & 16000 & 58 & $1.8 \times 10^{-3}$ & & 66 & $1.3 \times 10^{-3}$ & $1.7 \pm 0.1$
\end{tabular}

average $V_{\mathrm{w}}(\mathrm{PEO})$ was taken from the conventional Zimm plots and fixed at that value during the calculation. In this way an almost perfect agreement with the experimental data is obtained. Corresponding fit results are listed in Table 4 . The values obtained for $A_{2}$ are between $0.6 \times 10^{-3}$ and $2 \times 10^{-3} \mathrm{~cm}^{3} \mathrm{~mol} \mathrm{~g}^{-2}$, indicating good solvent conditions for PEO for all solvent mixtures. The second virial coefficients are plotted as a function of DMF content in Figure 8. For comparison, $A_{2}$ in pure water $\left(2.5 \times 10^{-3} \mathrm{~cm}^{3} \mathrm{~mol} \mathrm{~g}^{-2}\right)$ is also shown. This value was calculated from the $A_{2}-M_{\mathrm{w}}$ relationship determined by Devanand and Selser at $30{ }^{\circ} \mathrm{C} .{ }^{44}$ The plot qualitatively reveals characteristics similar to those found for the corona thickness, $D$, as a function of DMF.

Unfortunately, because of the limited number of data points and the experimental uncertainty involved, a detailed discussion of the change of solvent quality with increasing DMF content is not possible at the moment. Nevertheless, the main conclusion of this study is that on average a significant decrease of $A_{2}$ upon addition of DMF is observed, though the polymer always displays excluded-volume statistics. Furthermore, this drop in solvent quality for PEO leads to a sufficient destabilization of the PEP1-PEO20 micellar solutions such that block copolymer precipitates close to room temperature. It is interesting to note that the chain extension of the coronal PEO chains is observed to be sensitive to small changes in the solvent quality despite the low molar mass. Theoretically, $R_{\mathrm{g}}$ of low molar mass polymers in

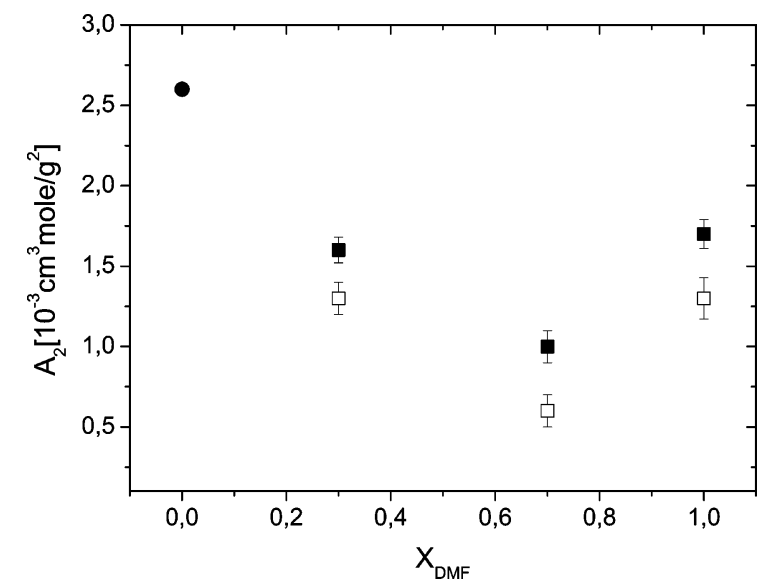

Figure 8. Second virial coefficient, $A_{2}$, vs DMF mole fraction, $X_{\mathrm{DMF}}$. The value for $A_{2}$ in pure water (filled circle at $X_{\mathrm{DMF}}=0$ ) was calculated from ref 44 . The other filled squares are from the conventional Zimm analysis and open squares from the generalized Zimm evaluation.

dilute solution should be nearly independent of the solvent quality provided that the solvent dissolves the polymer. ${ }^{45}$ Indeed, our results show that $R_{\mathrm{g}}$ is only weakly influenced in the solvent mixtures while the PEO corona shrinks significantly. Furthermore, the $R_{\mathrm{g}}$ found for the solvent mixtures agrees well with that in water using the scaling relation given by Devanand and Selser ${ }^{44}$ (approximately $62-65 \AA$ depending on the exact molar mass). Thus, we may conclude that the excludedvolume effects appear to be more effective in the corona.

\section{Conclusion}

The micelle formation of a highly asymmetric PEPPEO block copolymer may by effectively tuned by using water/DMF mixtures as selective solvent for PEO. The large difference in interfacial tension between PEP and water and PEP and DMF as well as the availability of well-defined polymers makes this system a perfect model system for fundamental studies of the influence of $\gamma$ on the aggregation behavior of block copolymers. The combined investigation of SANS and pendant drop tensiometry allowed to correlate aggregation numbers and interfacial tensions quantitatively. Because of the small differences in their predictions for the power law exponents, the obtained dependence of $P$ on $\gamma$ alone does not allow a distinction between the mean-field and scaling model. The observed starlike structure of the micelles - a manifestation of the scaling predictionstogether with $P$ vs $\gamma$ results consistently corroborates the scaling model. The reduction in solvent quality for PEO with increasing amount of DMF leads to a clear shrinkage of the micellar corona but, apparently, has no significant effect on the general aggregation behavior in this system.

It is worth mentioning that no important isotope effect could be detected. This conclusion can be drawn from the good agreement in the micellar characteristics for the two differently labeled polymers and, additionally, by almost identical values for the interfacial tensions of d-PEP1-OH and h-PEP1-OH in $\mathrm{D}_{2} \mathrm{O}$.

Presently, a thorough study on the role of interfacial tension for the exchange kinetics in block copolymer micelles is performed on the same system by timeresolved SANS. In a forthcoming paper it will be shown that $\gamma$ is the important quantity to vary the exchange rate of block copolymers between micelles. An important 
result of this study was that in pure water and in DMF/ water mixtures with small fractions of DMF the micelles are kinetically frozen. Because of this property and the adjustable structure, these micelles are currently used as tunable and kinetically stable model system for soft and ultrasoft colloids. ${ }^{46}$

Acknowledgment. We acknowledge financial support by the Deutsche Forschungsgemeinschaft. This work was partially performed in the framework of the Transregio Sonderforschungsbereich: Physics of Colloidal Dispersions in External Fields: Project A2.

\section{References and Notes}

(1) Hamley, I. W. The Physics of Block Copolymers; Oxford University Press: New York, 1998.

(2) Alexandridis, P.; Lindman, B. Amphiphilic Block Copolymers; Elsevier: Amsterdam, 2000.

(3) Tuzar, Z.; Kratochvil, P. Adv. Colloid Interface Sci. 1976, 6, 201.

(4) Tuzar, Z.; Kratochvil, P. In Surface and Colloid Science; Matijević, E., Ed.; Plenum Press: New York, 1993; Vol. 15, p 1.

(5) Riess, G.; Hurtrez, G.; Bahadur, P. In Encyclopedia in Polymer Science and Engineering, 2nd ed.; Mark, H. F., Bikales, N. M., Overberger, C. C., Menges, G., Eds.; WileyInterscience: New York, 1985; p 324.

(6) Riess, G. Prog. Polym. Sci. 2003, 28, 1107.

(7) Gast, A. P. Scientific Methods for the Study of Polymer Colloids and Their Applications; Kluwer Academic Publishers: Dordrecht, The Netherlands, 1990; p 311.

(8) Halperin, A.; Tirell, M.; Lodge, T. P. In Advances in Polymer Science; Schrag, J. L., Ed.; Springer-Verlag: Berlin,1992; Vol. 100, p 31.

(9) Yang, L.; Alexandridis, P. Langmuir 2000, 16, 4819

(10) Seo, Y. S.; Kim, M. W.; Ou-Yang, D. H.; Peiffer, D. G. Polymer $\mathbf{2 0 0 2}, 43,5629$

(11) Allgaier, J.; Poppe, A.; Willner, L.; Richter, D. Macromolecules 1997, 30, 1582.

(12) Poppe, A.; Willner, L.; Allgaier, J.; Stellbrink, J.; Richter, D. Macromolecules 1997, 30, 7463.

(13) Willner, L.; Poppe, A.; Allgaier, J.; Monkenbusch, M.; Lindner, P.; Richter, D. Europhys. Lett. 2000, 51, 628

(14) Kaya, H.; Willner, L.; Allgaier, J.; Stellbrink, J.; Richter, D. Appl. Phys. A 2002, 74 (Suppl), S499.

(15) Kaya, H. Ph.D. Thesis, Universität Münster, 2003.

(16) Willner, L.; Poppe, A.; Allgaier, J.; Monkenbusch, M.; Richter, D. Europhys. Lett. 2001, 55, 667.

(17) Leibler, L.; Orland, H.; Wheeler, J. C. J. Chem. Phys. 1983, 79,3550
(18) Noolandi, J.; Hong, K. M. Macromolecules 1983, 16, 1443.

(19) Nagarajan, R.; Ganesh, K. J. Chem. Phys. 1989, 90, 5843.

(20) Halperin, A. Macromolecules 1987, 20, 2943.

(21) Birshtein, T. M.; Zhulina, E. B. Polymer 1989, 30, 170.

(22) Izzo, D.; Marques, C. M. Macromolecules 1993, 26, 7189.

(23) deGennes, P.-G. Scaling Concepts in Polymer Physics; Cornell University Press: Ithaca, NY, 1979.

(24) Daoud, M.; Cotton, J. P. J. Phys. (Paris) 1982, 43, 531.

(25) Alexander, S. J. Phys. (Paris) 1977, 38, 977.

(26) deGennes, P.-G. J. Phys. (Paris) 1976, 37, 1443.

(27) Dozier, W. D.; Huang, J. S.; Fetters, L. J. Macromolecules 1991, 24, 2810.

(28) Pedersen, J. S.; Posselt, D.; Mortensen, K. J. Appl. Crystallogr. 1990, 23, 321.

(29) Beaucage, G. J. Appl. Crystallogr. 1995, 28, 717.

(30) Grest, G. S.; Fetters, L. J.; Huang, J. S.; Richter, D. In Advances in Chemical Physics; Prigogine, I., Rice, S. A., Eds.; Wiley: New York, 1996; Vol. 94, p 67.

(31) We can estimate the forward scattering of a blob according to $4 \pi \beta \mu \Gamma(\mu)=\phi V_{\mathrm{PEO}} X_{\mathrm{blob}}$. Here $X_{\mathrm{blob}}$ is the fraction of PEO in the blob. We then obtain $\beta \approx 3 \times 10^{-20.2 .5} \times 10^{-3} X_{\text {blob }}$ $\left(4 \pi 0.7 \Gamma(0.7) \approx X_{\text {blob }} \cdot 7 \times 10^{-24} \mathrm{~cm}^{3}\right.$, leaving the order of magnitude correct. For this calculation we have used $v=$ $0.588, \mu \approx 0.7$, and $\Gamma(0.7) \approx 1.3$.

(32) Willner, L.; Jucknischke, O.; Richter, D.; Roovers, J.; Zhou, L.-L.; Toporowski, P. M.; Fetters, L. J.; Huang, J. S.; Lin, M. Y.; Hadjichristidis, N. Macromolecules 1994, 27, 3821.

(33) Devanand, K.; Selser, J. C. Nature (London) 1990, 343, 739

(34) Woodley, D. M.; Dam, C.; Lam, H.; LeCave, M.; Devanand, K.; Selser, J. C. Macromolecules 1992, 25, 5283.

(35) Pedersen, J. S.; Svaneborg, C. Curr. Opin. Colloid Interface Sci. 2002, 7, 158

(36) Castelletto, V.; Hamley, I. W. Curr. Opin. Colloid Interface Sci. 2002, 7, 167.

(37) Svaneborg, C.; Pedersen, J. S. J. Chem. Phys. 2000, 112, 9661.

(38) Svaneborg, C.; Pedersen, J. S. Phys. Rev. E 2001, 64, 010802.

(39) Svaneborg, C.; Pedersen, J. S. Macromolecules 2002, 35, 1028.

(40) Won, Y.-Y.; Davis, H. T.; Bates, F. S.; Agamalian, M.; Wignall, G. D. J. Phys. Chem. 2000, 104, 7134.

(41) Smith, G. D.; Yoon, D. Y.; Jaffe, R. L.; Colby, R. H.; Krishnamoorti, R.; Fetters, L. J. Macromolecules 1996, 29, 3462 .

(42) Vennemann, N.; Lechner, M. D.; Oberthür, R. C. Polymer 1987, 28, 1738.

(43) Sommer, C.; Pedersen, J. S.; Stein, P. C. J. Phys. Chem. B 2004, 108, 6242 .

(44) Devanand, K.; Selser, J. C. Macromolecules 1991, 24, 5943.

(45) Weill, G.; des Cloizeaux, J. J. Phys. (Paris) 1979, 40, 99.

(46) Physics of Colloidal Dispersions in External Fields, Transregio Sonderforschungsbereich der Deutschen Forschungsgemeinschaft: Project A2: Frozen Block Copolymer Micelles as "tunable" Model System for Soft Colloids.

MA035633N 